\title{
Complicated Tubercular Pericarditis Presenting as Ventricular Apical Aneurysm
}

\author{
Sonali Sethi ${ }^{1 *}$ and SatyajitGodhi ${ }^{2 *}$ \\ ${ }^{1}$ Department of Radiology, Prima Diagnostics, Bangalore, Karnataka, India \\ ${ }^{2}$ Consultant Surgical Gastroenterology, Apollo Hospitals, Bangalore, Karnataka, India
}

*Corresponding author: Sonali Sethi, Consultant Radiologist, Prima Diagnostics, Bangalore, Karnataka, India Satyajit Godhi, Consultant Surgical Gastroenterology, Apollo Hospitals, Bangalore, Karnataka, India

Submission: 侮 December 05, 2017; Published: 毕 December 15, 2017

\begin{abstract}
A 25 year old female presented with features of right heart failure. She was treated for pulmonary tuberculosis in the past and completed treatment four years back. Contrast enhanced CT scan of the thorax was performed which revealed chronic calcific peri-carditis with a narrow necked left ventricular apical free wall aneurysm (Figure 1a \& 1b).
\end{abstract}

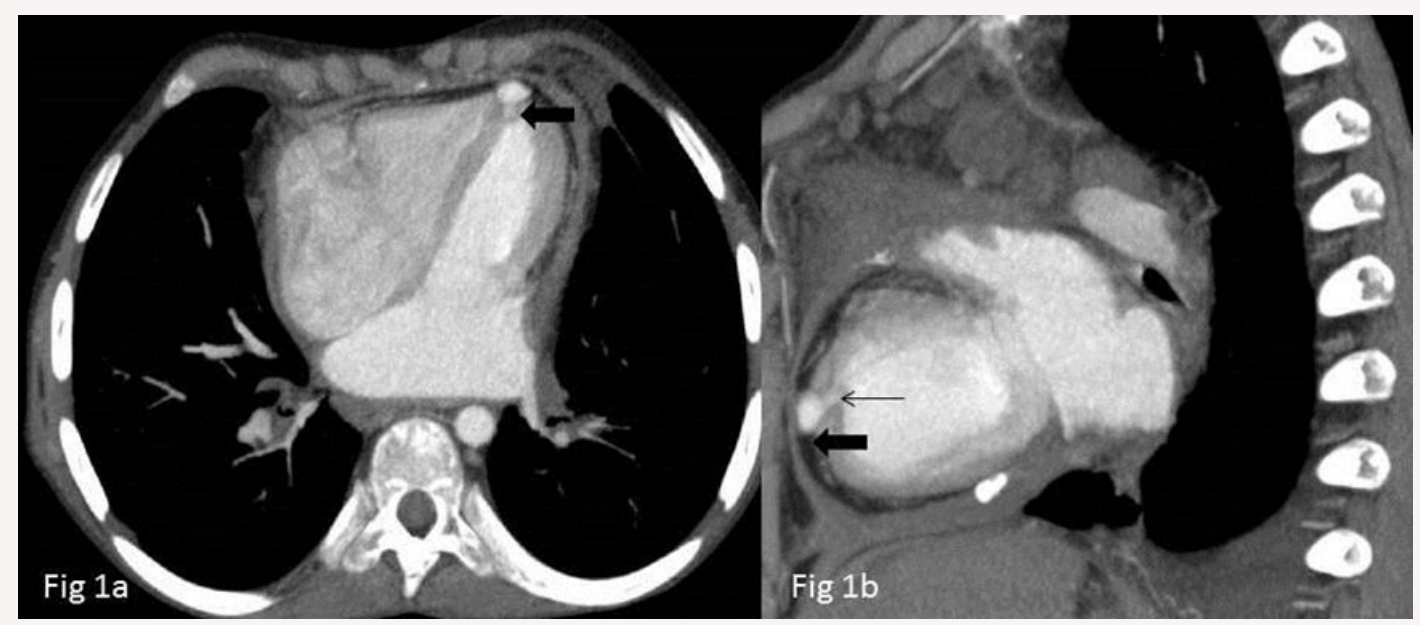

Figures 1a \& 1b: Contrast enhanced axial and sagittal reformatted images of thorax.

Small contrast filled out-pouching involving the ventricular apex (bold black arrow) showing a narrow neck (thin black arrow). Reduced size of ventricles with prominence of both atria is seen.

\section{Introduction}

Tuberculosis is a common cause of mortality and morbidity in the developing countries. Several fatal complications of tuberculosis have been described; however cardiac pseudo aneurysm is a rare and fatal complication. The recognition of this entity by the radiologist and cardiologist is important for proper and timely surgical management.

\section{Case Report}

A 27 year old female presented with complaints of breathlessness, fever and easy fatigability. She was treated for pulmonary tuberculosis in the past and completed treatment four years back and had been asthenic since then. She belonged to a poor socio-economic status. Her two children also suffered from pulmonary tuberculosis and had received treatment for the same; however the younger one could not survive and died of severe hemoptysis three years back.

On examination she was pale and had increased jugular venous pressure and pedal edema. Lab investigations revealed microcytic hypochromic anaemia with haemoglobin of $6 \mathrm{gm} / \mathrm{dL}$. A Contrast enhanced CT scan of the thorax was performed which revealed thickened pericardium with areas of dense calcification (Figure 2a \& $2 b)$. There was bi-atrial prominence with relatively reduced volume 
of ventricles and dilated IVC consistent with constrictive pericarditis. Incidentally a patent ventricular apical pseudo aneurysm was demonstrated having a narrow neck arising from the left ventricular apical free wall (Figure 3a \& 3b). Calcified mediastinal nodes were present along with fibrotic changes in bilateral lung apices with no radiological evidence of active tuberculosis.

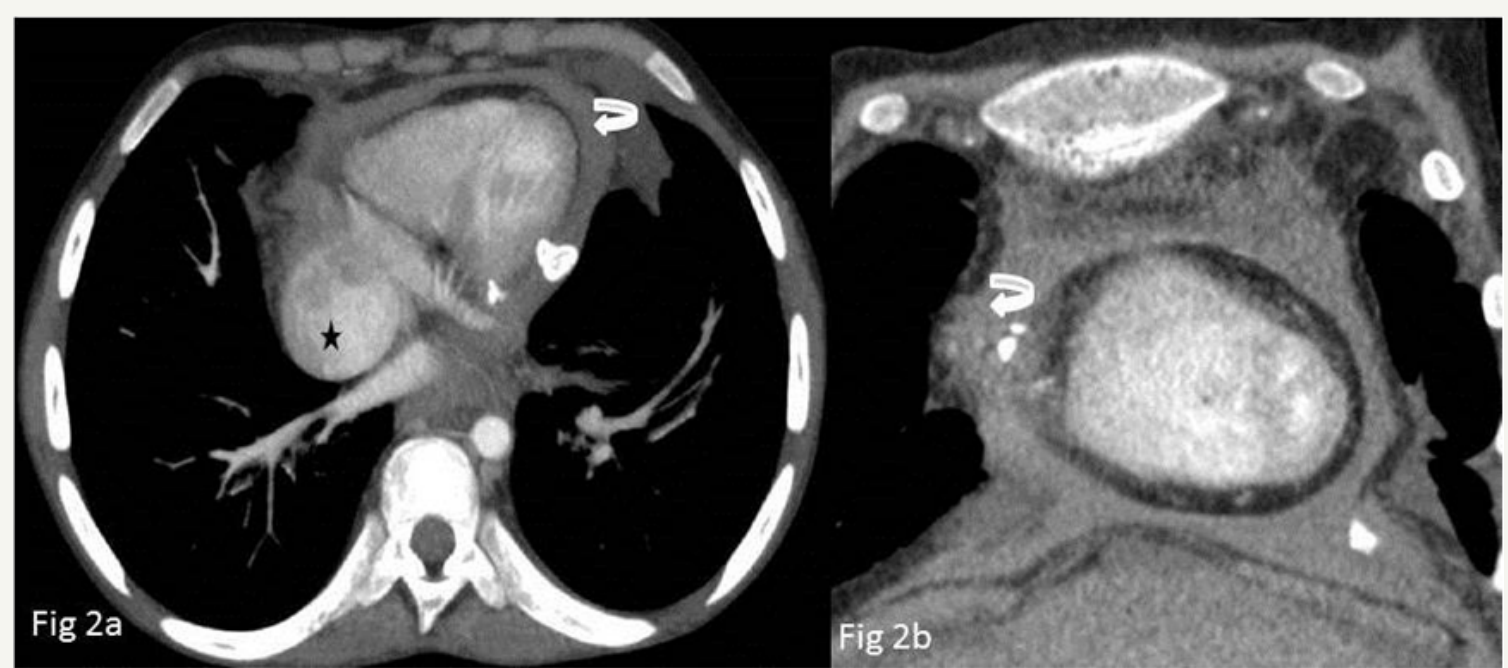

Figure 2: Thickened pericardium with large areas of calcification and reduced size of ventricles (curved white arrow). The IVC is dilated (black star).

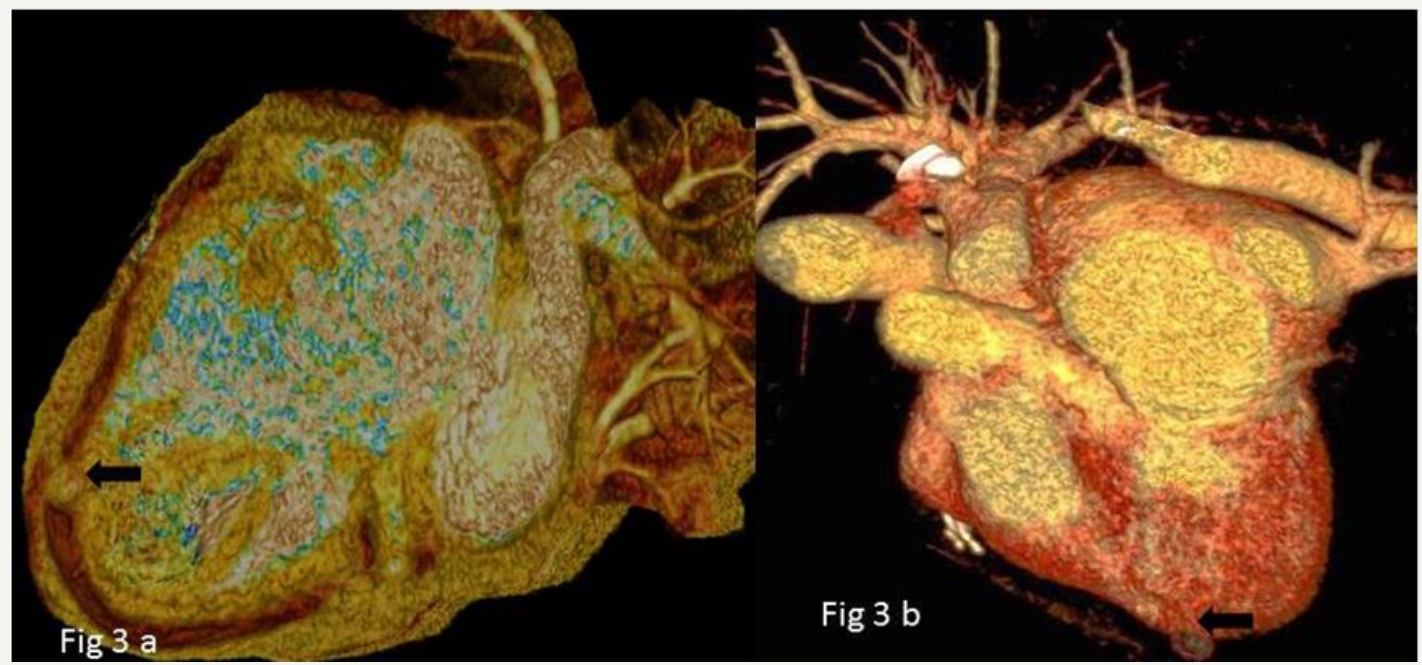

Figure 3: Volume rendered images showing a nodular projection at the ventricular apex (bold black arrow).

The echo cardio graphic features were consistent with chronic constrictive peri-carditis, the aneurysm could however not be seen on trans-thoracic echocardiography.

The patient was explained the risks and complications of ventricular pseudo-aneurysm like rupture and thrombosis with consequent embolism and was counselled for surgical pericardiectomy and excision of the pseudo-aneurysm. She however refused surgery due to her economic condition.

\section{Discussion}

Cardiac involvement in tuberculosis is most commonly in the form of pericarditis due to contiguous lymph node involvement or haematogenous spread. It may result in pericardial effusion and pericardial thickening with or with-out calcification in chronic stages.

Myocardial involvement is less common and more fatal with reported complications in the form of arrhythmias, complete heart block with sudden cardiac death, right ventricular outflow tract obstruction, aortic regurgitation and superior vena cava obstruction [1]. It may occur in the form of diffuse myocarditis, localized myocarditis or disseminated military lesions [1]. Endocardial involvement has been described in the form of tubercular valvular and endocardial vegetation's [2].

Ventricular aneurysms have been rarely described in association with tubercular pericarditis in the form of isolated case reports [1,3-5]. Majority of the ventricular aneurysms are caused 
by post myocardial infarction. Other causes are post-surgical, post traumatic and post infective. Rare causes are alpha 1 antitrypsin deficiency, tumor infiltration and behcet disease [5].

The patho physiology of development of pseudo-aneurysms in tuberculosis is secondary to contained rupture of infected soft myocardial tuberculomas coupled with hemodynamic strain with overlying thickened fibrous pericardium [5].

The majority of cases had non-specific presentation and the aneurysm was discovered on imaging. The diagnosis of tubercular etiology was made in most of the cases on post-operative biopsy. The diagnosis of tubercular pseudo-aneurysm was made by us based on the imaging findings. It is imperative for the radiologist to be abreast with the existence of this entity and it's imaging findings for timely surgical management.

\section{References}

1. Halim MA, Mercer EN, Guinn GA (1985) Myocardial tuberculoma with rupture and pseudo aneurysm formation-successful surgical treatment. Br Heart J 54(6): 603-04.

2. Sultan FA, Fatimi S, Jamil B, Moustafa SE, Mookadam F (2010) Tuberculous endocarditis: valvular and right atrial involvement. Eur J Echocardiogr 11(4): E13.

3. Palweni WC, Harrisberb RJ, Levin ES (1994) Successful surgery for a tuberculous left ventricular aneurysm in a child. Cardiology in the Young 4: 399-401

4. Masoumi M, Solgy G (2002) Pseudoaneurysm of right ventricle due to localized tuberculosis of myocardium: A Case Report Med J Islam Repub Iran 16(1): 47-49.

5. Wei W, Hui C, Sheng-Yu W, Xin D (2014) Uncommon cause of left ventricular pseudoaneurysm: case report and review of literature. Thorac Cardiovasc Surg 62(4): 375-377. 